\title{
ALMOST FACTORISABLE INVERSE SEMIGROUPS
}

\author{
by M. V. LAWSON
}

(Received 22 July, 1992)

0. Introduction. In [3], McAlister introduced a class of semigroups, called covering semigroups, which were shown to play an important role in the theory of $E$-unitary covers of semigroups. Strangely, this class of semigroups appears to have received little attention subsequently. It is the aim of this paper to rehabilitate them and to study their properties in more detail. As a first step, we have chosen to rename them almost factorisable semigroups, since they can be regarded as the semigroup analogues of factorisable inverse monoids. Before discussing the contents of this paper in more detail we recall some standard terminology.

We assume the reader is familiar with basic inverse semigroup theory. For convenience, however, we describe here the notation and terminology used which is not explicitly defined in the text. All semigroups will be inverse. The (generalised) inverse of an element $x$ will be denoted by $x^{-1}$. The set of idempotents of a semigroup is written $E(S)$; if $A \subseteq S$ then $E(A)=A \cap E(S)$. The natural partial order is denoted by $\leqslant$. A semigroup $S$ is $E$-unitary if $e \leqslant x$ and $e \in E(S)$ implies $x \in E(S)$. An $E$-unitary cover of an inverse semigroup $S$ is an $E$-unitary semigroup $P$ and a surjective, idempotent separating homomorphism $\theta: P \rightarrow S$. It is well-known that every inverse semigroup has an $E$-unitary cover [7]. If $S$ is a monoid then the group of units of $S$ will be denoted by $U(S)$. An inverse monoid $S$ is said to be factorisable if for each $s \in S$ there exists $g \in U(S)$ such that $s \leq g$. The minimum group congruence $\sigma$ on a semigroup $S$ is defined by $x \sigma y$ if, and only if, there exists $z \in S$ such that $z \leq x$ and $z \leq y$. If $(Q, \leq)$ is a poset then $A \subseteq Q$ is said to be an order ideal if $b \leq a \in A$ implies that $b \in A$. The set $[a]=\{b \in Q: b \leq a\}$ is called a principal order ideal. The trace product of $x$ and $y$ in a semigroup $S$ is defined to be $x y$ if $x^{-1} x=y y^{-1}$, and undefined otherwise. If $A$ and $B$ are sets then the projections from $A \times B$ to $A$ and $B$ respectively are denoted by $\pi_{1}$ and $\pi_{2}$. If $\theta: A \rightarrow B$ is a function then $\operatorname{ker} \theta$ denotes the equivalence relation induced on $A$. Finally, if $\rho$ is a congruence then $\rho^{4}$ denotes the corresponding natural map.

The paper is divided into three sections. In Section 1, we discuss the basic properties of almost factorisable inverse semigroups and show that they are very closely related to factorisable inverse monoids. Specifically, we prove in Proposition 1.7 that an inverse monoid is almost factorisable if, and only if, it is factorisable. In Theorem 1.10, we show that for every almost factorisable inverse semigroup $S$ there is a factorisable inverse monoid $F$ such that $S$ is isomorphic to $F \backslash U(F)$. In Section 2, we describe all strongly $E$-unitary covers of almost factorisable inverse semigroups. This is achieved in Proposition 2.3 and Theorem 2.5. In Theorem 2.4, we show that the class of almost factorisable semigroups is the closure under homomorphisms of the class of strongly $E$-unitary semigroups. In the final section, Section 3, we relate arbitrary inverse semigroups to almost factorisable inverse semigroups. The main result, Theorem 3.8, shows that every $E$-unitary cover of an inverse semigroup $S$ over a semilattice is associated with an embedding of $S$ into an almost factorisable inverse semigroup $F$. The embedding of $S$ into $F$ is of a special kind which we call an enlargement. We conclude the paper with a reformulation of a question posed by Henkell and Rhodes [9]: we show that an inverse 
monoid $S$ has a finite $F$-inverse cover if, and only if, $S$ has a finite, almost factorisable enlargement.

1. Basic properties of almost factorisable semigroups. McAlister's original definition of almost factorisable semigroups was couched in terms of one-to-one partial right translations. We prefer to work instead with the theory of permissible subsets, introduced by Schein [10]. It is well-known (see Petrich [7, Theorem V.2.8]) that the semigroup of all one-to-one partial right translations is isomorphic to the semigroup of permissible subsets, but permissible subsets appear to us to be easier to work with. We begin therefore by recalling their properties.

Definition. Two elements $x$ and $y$ in an inverse semigroup $S$ are said to be compatible, written $x \sim y$, if both $x y^{-1}$ and $x^{-1} y$ are idempotents. A subset $A$ of $S$ is said to be compatible if the elements of $A$ are pairwise compatible.

It is easy to see that the compatibility relation is reflexive and symmetric.

LemmA 1.1. Let $a \sim b$ and either $b^{-1} b \leq a^{-1} a$ or $b b^{-1} \leq a a^{-1}$. Then $b \leq a$.

Proof. Suppose that $a \sim b$ and $b^{-1} b \leq a^{-1} a$. From $b^{-1} b \leq a^{-1} a$ we obtain $b b^{-1} b \leq$ $b a^{-1} a$, and so $b \leq\left(b a^{-1}\right) a$. But $a \sim b$ implies that $b a^{-1}$ is an idempotent and so $(b a)^{-1} a \leq a$. Hence $b \leq a$. The other case is proved similarly.

We summarise below the most important properties of the compatibility relation; they are all well-known and are included for the sake of completeness.

THEOREM 1.2. (i) The compatibility relation is stable with respect to left and right multiplication.

(ii) The compatibility relation is contained in the minimum group congruence $\sigma$.

(iii) The compatibility relation is transitive if, and only if, it equals $\sigma$.

(iv) The semigroups in which $\sim$ and $\sigma$ coincide are just the E-unitary semigroups.

(v) $x \sim y$ if, and only if, $x$ and $y$ have a meet $x \wedge y=z$ with respect to the natural partial order, such that $z^{-1} z=x^{-1} x y^{-1} y$ and $z z^{-1}=x x^{-1} y y^{-1}$. Furthermore, if $x \sim y$ then

$$
x \wedge y=x x^{-1} y=y y^{-1} x=x y^{-1} y=y x^{-1} x=y x^{-1} y=x y^{-1} x .
$$

Proof. (i) Straightforward.

(ii) Let $a \sim b$. Then $a^{-1} b=e$ an idempotent. Hence $\sigma\left(a^{-1} b\right)=\sigma(e)$, and so $\sigma(b)=\sigma(a)$, since $\sigma(e)$ is the identity of $S / \sigma$.

(iii) If $\sim$ is equal to $\sigma$ then it is clearly transitive. Conversely, suppose that $\sim$ is transitive. Then by (i), $\sim$ is a congruence. Indeed, it is a group congruence since any two idempotents are compatible. But by (ii), $\sim$ is contained in $\sigma$, and so is equal to $\sigma$, since $\sigma$ is the minimum group congruence.

(iv) Suppose that $S$ is $E$-unitary and that $a \sigma b$. Then $a^{-1} b \sigma b^{-1} b$, and so there exists $z \leq a^{-1} b, b^{-1} b$. Thus $z$ is an idempotent and, since $S$ is $E$-unitary, $a^{-1} b$ is an idempotent. Similarly, $a b^{-1}$ is an idempotent. It follows that $a \sim b$. Conversely, suppose that $\sim$ is equal to $\sigma$ and that $e \leq x$. Clearly $\sigma(e)=\sigma(x)$. Thus $\sigma\left(x^{-1} x\right)=\sigma(x)$, and so by assumption we have that $x^{-1} x \sim x$. We can now apply Lemma 1.1, to obtain $x=x^{-1} x$.

(v) Suppose that $x \sim y$. Put $z=x x^{-1} y$. Clearly, $z \leq y$. But since $x \sim y, x^{-1} y$ is an 
idempotent so that $z \leq x$. Now let $w \leq x, y$. Then $w w^{-1} \leq x x^{-1}$ so that $w=w w^{-1} w \leq$ $x x^{-1} y=z$. Thus $z=x \wedge y$. Similar arguments show that

$$
x x^{-1} y=y y^{-1} x=x y^{-1} y=y x^{-1} x .
$$

Since $x^{-1} y$ is an idempotent we have that $y^{-1} x=x^{-1} y$. Thus

$$
x x^{-1} y=x y^{-1} x \text { and } y y^{-1} x=y x^{-1} y .
$$

Finally,

$$
z^{-1} z=\left(x y^{-1} y\right)^{-1} x y^{-1} y=y^{-1} y x^{-1} x \quad \text { and } \quad z z^{-1}=x x^{-1} y\left(x x^{-1} y\right)^{-1}=x x^{-1} y y^{-1} .
$$

Conversely, suppose that $x$ and $y$ have a meet $z$ such that $z^{-1} z=x^{-1} x y^{-1} y$ and $z z^{-1}=x x^{-1} y y^{-1}$. From the definition of the natural partial order, $z=x z^{-1} z$ and $z=z z^{-1} y$. Thus $z=x y^{-1} y=x x^{-1} y$, and so $x^{-1} x y^{-1} y=x^{-1} y$, an idempotent. Similarly, $x y^{-1}$ is an idempotent and so $x \sim y$.

Definition. A permissible subset $A$ of $S$ is a compatible order ideal. The set of all permissible subsets is denoted by $C(S)$.

LEMMA 1.3. If $A$ is a permissible subset of $S$ and $a, b \in A$ and either $a^{-1} a=b^{-1} b$ or $a a^{-1}=b b^{-1}$ then $a=b$.

Proof. Immediate from Lemma 1.1 and the definition of a permissible subset.

$C(S)$ is an inverse monoid under the usual multiplication of subsets. The set of idempotents is just the set of order ideals contained in $E(S)$, the identity is $E(S)$, the natural partial order is subset inclusion, and the inverse of $A$ is $A^{-1}$ (see Petrich [7] and Schein [10] for details). The semigroup $S$ is embedded in $C(S)$ by means of the homomorphism $\iota: S \rightarrow C(S)$ defined by $\iota(s)=[s]$. The group of units of $C(S)$ is denoted by $\Sigma(S)$.

The following is due to Nambooripad [6].

LEMMA 1.4. Let $S$ be an inverse semigroup in which $z \leq x_{1} \ldots x_{n}$. Then there exist elements $y_{i}$ such that $y_{i} \leq x_{i}, z=y_{1} \ldots y_{n}$ and $y_{1} \ldots y_{n}$ is a trace product.

Definition. Let $A$ be a subset of an inverse semigroup $S$. Then $[A]$ is the order ideal generated by $A$.

Definition. A homomorphism $\theta: S \rightarrow T$ is said to be full if $E(T) \subseteq \theta(S)$.

The following results are well-known.

Proposition 1.5. (i) If $A$ is a compatible subset of $S$ and $\theta$ is a homomorphism from $S$ to $T$ then $\theta(A)$ is a compatible subset of $T$.

(ii) If $A$ is a compatible subset in $S$, then [A] is a permissible subset of $S$.

(iii) If $\theta: S \rightarrow T$ is a homomorphism then $C(\theta): C(S) \rightarrow C(T)$ defined by $C(\theta)(A)=$ $[\theta(A)]$ is a homomorphism which preserves arbitrary joins of compatible subsets.

(iv) If $\theta: S \rightarrow T$ is a full homomorphism then $\theta: \Sigma(S) \rightarrow \Sigma(T)$ is a well-defined homomorphism. 
Proof. (i) Let $\theta(a), \theta(b) \in \theta(A)$. Then $\theta(a)^{-1} \theta(b)=\theta\left(a^{-1} b\right)$. By assumption $a$ and $b$ are compatible and so $a^{-1} b$ is an idempotent. Thus $\theta(a)^{-1} \theta(b)$ is an idempotent. Similarly, $\theta(a) \theta(b)^{-1}$ is an idempotent.

(ii) By definition $[A]$ is an order ideal, so it only remains to check that it is a compatible subset. Let $x, y \in[A]$. Then there exist elements $a, b \in A$ such that $x \leq a$ and $y \leq b$. By assumption $a$ and $b$ are compatible. Thus $a^{-1} b$ and $a b^{-1}$ are idempotents. Consequently, $x^{-1} y$ and $x y^{-1}$ are idempotents, and so $x \sim y$, as required.

(iii) That $C(\theta)$ is well-defined is immediate from (i) and (ii). To show that $C(\theta)$ is a homomorphism we have to show that $[\theta(A B)]=[\theta(A)][\theta(B)]$ for all $A, B \in C(S)$. It is clear that $[\theta(A)][\theta(B)] \subseteq[\theta(A B)]$. Let $x \in[\theta(A B)]$. Then $x \leq \theta(a b)=\theta(a) \theta(b)$ some $a \in A$ and $b \in B$. But by Lemma 1.4, there exist $u \leq \theta(a)$ and $v \leq \theta(b)$ such that $x=u v$. But $u v \in[\theta(A)][\theta(B)]$, and so $x \in[\theta(A)][\theta(B)]$. To show that $C(\theta)$ preserves arbitrary joins of compatible subsets, we have to show that for any compatible subset $\left\{A_{i}: i \in I\right\}$ of $C(S)$ we have that $\left[\theta\left(\cup A_{i}\right)\right]=\cup\left[\theta\left[A_{i}\right)\right]$; this is straightforward to check.

(iv) Let $A \in \Sigma(S)$. We claim that $C(\theta)(A)=\theta(A)$ and that $\theta(A) \in \Sigma(T)$. It is immediate that $\theta(A) \subseteq[\theta(A)]$. Let $x \leq \theta(a)$ where $a \in A$. Now $\theta$ is a full homomorphism, and so there exists $e \in E(S)$ such that $\theta(e)=x^{-1} x$. But then $\theta(a e)=\theta(a) \theta(e)=x$. Thus $x \in \theta(A)$. Finally, since $\theta$ is full, $E(S)$ is mapped to $E(T)$. But $E(S)$ is the identity of $C(S)$ and $E(T)$ is the identity of $C(T)$. Thus the units of $C(S)$ are mapped to the units of $C(T)$.

We can now define the class of semigroups with which this paper is concerned.

Definition. An inverse semigroup $S$ is said to be almost factorisable if for each $x \in S$ there exists $A \in \Sigma(S)$ such that $x \in A$.

LEMMA 1.6. Let $S$ be an almost factorisable inverse monoid. Then

(i) every left invertible element is invertible;

(ii) every right invertible element is invertible.

Proof. We prove (i), the proof of (ii) is similar. Let $g \in S$ such that $g^{-1} g=1$. By definition $g \in A$ for some $A \in \Sigma(S)$. But $A A^{-1}=E(S)$, and so there exists $h \in A$ such that $h h^{-1}=1$. But $h \sim g$ and $g g^{-1} \leq h h^{-1}$ and so by Lemma 1.1, we have that $g \leq h$. Thus $g=h g^{-1} g$, and so $g=h$.

The following result is essentially Proposition 4.11 of [3].

Proposition 1.7. An inverse monoid $S$ is almost factorisable if, and only if, it is factorisable.

Proof. Let $S$ be an almost factorisable inverse monoid, and let $x$ be any element of $S$. Then $x \in A$ for some $A \in \Sigma(S)$. Since $E(S)=A^{-1} A$ there exists an element $g \in A$ such that $g^{-1} g=1$. But, being left invertible, $g$ is invertible by Lemma 1.6. Furthermore, by Lemma $1.1, x \leq g$. Hence $S$ is factorisable. Conversely, suppose that $S$ is factorisable. Then for each $x \in S$ there exists an invertible element $g$ such that $x \leq g$, and so $x \in[g]$. It is easy to check that $[g] \in \Sigma(S)$. Hence $S$ is almost factorisable.

It is worth making explicit a result anticipated in the proof of Proposition 1.7.

Lemma 1.8. Let $F$ be a factorisable inverse monoid. Then $\Sigma(F)=\{[g]: g \in U(F)\}$. 
Proof. It is clear that if $g \in U(F)$ then $[g] \in \Sigma(F)$. Now let $A \in \Sigma(F)$. Since $1 \in A^{-1} A$, there exists $g \in A$ such that $g^{-1} g=1$. By Lemma 1.6, $g$ is invertible. Let $a \in A$. Then $a \sim g$ and $a^{-1} a \leq g^{-1} g$. Thus by Lemma 1.1, $a \leq g$. It follows that $A=[g]$.

DEFINITION. Let $S$ be an inverse semigroup and let $e$ and $f$ be idempotents such that $e \leq f$. Define a function $\psi_{f, e}: L_{f} \rightarrow L_{e}$ by $\psi_{f, e}(x)=x e$. The family of functions $\left\{\psi_{f, e}: e \leq f\right.$, $e, f \in E(S)\}$ is called the set of structure mappings of $S$. See Petrich [7] for more information.

Given an inverse semigroup, perhaps by means of a Cayley table, it does not appear to be easy to determine whether it is almost factorisable or not. In the proposition below we provide some necessary conditions.

Proposition 1.9. (i) Let $S$ be almost factorisable.

(a) For each $x \in S$ and $e \in E(S)$ there exists $y \in S$ such that $y^{-1} y=e$ and $x \sim y$.

(b) For each $x \in S$ and $e \in E(S)$ there exists $y \in S$ such that $y y^{-1}=e$ and $x \sim y$.

(ii) In any semigroup, (i)(a) and (i)(b) are equivalent.

(iii) A semigroup satisfying (i)(a) has surjective structure mappings.

(iv) Let $S$ be a semigroup which satisfies (i)(a) and has a set of maximal elements $M$. Then

(a) $x \in M$ if and only if $x^{-1} x$ is a maximal idempotent,

(b) $x \in M$ if and only if $x x^{-1}$ is a maximal idempotent,

(c) $x \in M$ if and only if $x \mathscr{D} e$ and $e$ is a maximal idempotent.

Proof. (i)(a) Let $x \in S$ and $e \in E(S)$. Then $x \in A$ some $A \in \Sigma(S)$. But $A^{-1} A=E(S)$ so that there exists $y \in A$ such that $y^{-1} y=e$ and $x \sim y$.

(b) The proof is similar to that of (a).

(ii) Suppose (i)(a) holds. Let $x$ be any element and $e$ any idempotent. By (i)(a), there exists an element $z$ such that $z^{-1} z=e$ and $x^{-1} \sim z$. But $x^{-1} \sim z$ implies that $x \sim z^{-1}$. Put $y=z^{-1}$. Then $y y^{-1}=e$ and $x \sim y$. Thus (i)(b) holds. A similar argument shows that (i)(b) implies (i)(a).

(iii) Consider the structure mapping $\psi_{f . e}: L_{f} \rightarrow L_{e}$ and let $x \in L_{e}$. By (i)(a) there exists $y \in S$ such that $y^{-1} y=f$ and $y \sim x$. By Lemma 1.1, $x \leq y$ and so $\psi_{f, e}(y)=x$.

(iv)(a) Let $e$ be a maximal idempotent and let $x$ be any element such that $x^{-1} x=e$. Suppose that $x \leq y$. Then $e=x^{-1} x \leq y^{-1} y$. But $e$ is maximal and so $x=y$, and $x$ is maximal. Now let $x$ be maximal. Suppose that $x^{-1} x \leq e$, where $e$ is an idempotent. By (i)(a), there exists $y \in S$ such that $y^{-1} y=e$ and $x \sim y$. By Lemma 1.1, this means that $x \leq y$. Thus $x=y$, since $x$ is maximal, and so $x^{-1} x=e$. Hence $x^{-1} x$ is a maximal idempotent.

(b) The proof of this case is similar to that of (a).

(c) Suppose that $x \mathscr{D} e$ where $e$ is a maximal idempotent. Then $x \mathscr{R} y \mathscr{L} e$ for some element $y$ in $S$. But $e$ a maximal idempotent implies by (a) above that $y$ is a maximal element, and by (b) above that $x$ is a maximal element. The converse is immediate by (a).

ExAmple. The condition (i)(a) above is not a sufficient condition for almost factorisability; the Munn semigroups of the semilattices $E$ and $F$ in Figure 1 satisfy (i)(a) but neither is almost factorisable. (I am grateful to Tom Hall for these examples.)

ExAmples. (i) Let $F$ be a factorisable inverse monoid with group of units $G$. Then $F^{\prime}=F \backslash G$ is an almost factorisable inverse semigroup. 


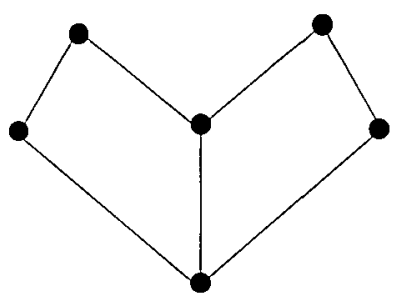

E

Figure 1.

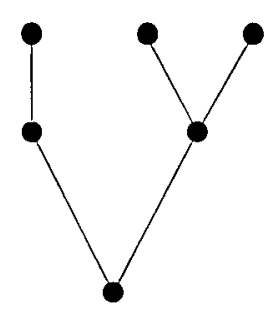

$\mathrm{F}$

(ii) Let $S$ be the semidirect product of a group $G$ and a meet semilattice $Y$. Thus $S=Y \times G$ with multiplication given by

$$
(x, g)(y, h)=(x \wedge g y, g h) .
$$

$S$ is an inverse semigroup in which the inverse of $(x, g)$ is $\left(g^{-1} x, g^{-1}\right)$, the natural partial order is defined by $(x, g) \leq(y, h)$ iff $g=h$ and $x \leq y$, and $(x, g) \sigma(y, h)$ iff $g=h$. It is easy to see that $S$ is $E$-unitary and so $\sigma$ coincides with the compatibility relation. The elements of $C(S)$ are thus the subsets of $Y \times G$ of the form $Y^{\prime} \times\{g\}$ where $Y^{\prime}$ is an order ideal of $Y$ and $g$ is an element of $G$. It follows that the elements of $\Sigma(S)$ are the subsets of $S$ of the form $Y \times\{g\}$. It is now clear that $S$ is almost factorisable. Adjoin a maximum element 1 to the meet semilattice $(Y, \leq)$ and denote the resulting semilattice by $\left(Y^{\prime}, \leq\right)$. The action of $G$ on $Y$ may be easily extended to $Y^{\prime}$ by defining g.1 1 for all $g \in G$. The semigroup $F=Y^{\prime} \times G$ is factorisable and $F \backslash U(F)=S$.

Definition. A semigroup isomorphic to a semidirect product of a group and a meet semilattice will be called strongly E-unitary.

Examples (i) and (ii) above motivate the following.

THEOREM 1.10. (i) Let $S$ be an almost factorisable semigroup and $F$ a factorisable inverse monoid such that $F \backslash U(F)=S$. Then there is a group homomorphism $\alpha: U(F) \rightarrow$ $\Sigma(S)$ such that $\cup \alpha(U(F))=S$.

(ii) Let $S$ be an almost factorisable semigroup and $\alpha: G \rightarrow \Sigma(S)$ a group homomorphism such that $\cup \alpha(G)=S$. Then there exists a factorisable inverse monoid $F$ with group of units $G$ such that $F \backslash G$ is isomorphic to $S$.

Proof. (i) We show first that $\{[g] \backslash\{g\}: g \in U(F)\} \subseteq \Sigma(S)$. Let $A=[g] \backslash\{g\}$ where $g \in U(F)$. It is clear that $A$ is a permissible subset of $S$. Let $e$ be any idempotent of $S$. Then $a=g e \in A$ and $a^{-1} a=e$. Thus $A^{-1} A=E(S)$. A similar argument shows that $A A^{-1}=E(S)$. Now define $\alpha: U(F) \rightarrow \Sigma(S)$ by $\alpha(g)=[g] \backslash\{g\}$. Is it clear that $\alpha$ is well-defined and that $U \alpha(U(F))=S$. It remains only to show that $\alpha$ is a homomorphism. Thus we need to show that $[g h] \backslash\{g h\}=([g] \backslash\{g\})([h] \backslash\{h\})$. Let $x \leq g$ and $y \leq h$. Suppose that $x y=g h$. Then $y^{-1} x^{-1} x y=1$. But $y^{-1} x^{-1} x y \leq y^{-1} y$. Hence by Lemma 1.6, $y=h$. Similarly, $x=g$. Hence $([g] \backslash\{g\})([h] \backslash\{h\}) \subseteq[g h] \backslash\{g h\}$. Now let $x \leq g h$. By Lemma 1.4, there are elements $u$ and $v$ such that $x=u v$ where $u \leq g, v \leq h$ and $u v$ is a trace product. Suppose that $u=g$. Then $x x^{-1}=1$ and so by Lemma 1.6, $x=g h$. Similarly, if $v=h$ then $x=g h$. Hence $[g h] \backslash\{g h\} \subseteq([g] \backslash\{g\})([h] \backslash\{h\})$. 
(ii) We begin by proving the theorem for the special case where $\alpha$ is the identity map on $\Sigma(S)$. Put $F^{\prime}=\{[s]: s \in S\} \cup \Sigma(S)$. We show first that $F^{\prime}$ is an inverse subsemigroup of $C(S)$. It is clear that $F^{\prime}$ is closed under inversion. Consider the product $[s] A$ where $A \in \Sigma(S)$. Since $s^{-1} s \in E(S)=A A^{-1}$ there exists an element $a \in A$ such that $s^{-1} s=a a^{-1}$. We claim that $[s] A=[s a]$. Let $x \in[s a]$. Then $x \leq s a$. Thus by Lemma 1.4, there exist elements $u$ and $v$ such that $x=u v, u \leq s$ and $v \leq a$. It follows that $x \in[s] A$. Now let $x \in[s] A$. Then $x=t b$ where $t \leq s$ and $b \in A$. Now $t b=t\left(t^{-1} t b\right)$. Since $A$ is an order ideal $t^{-1} t b \in A$. But

$$
t^{-1} t b\left(t^{-1} t b\right)^{-1}=t^{-1} t b b^{-1} \leq s^{-1} s=a a^{-1} .
$$

Thus by Lemma 1.1, we have that $t^{-1} t b \leq a$. Thus $t b \leq s a$, as required. Similarly, $A[s]=[a s]$, where $a \in A$ is such that $a^{-1} a=s s^{-1}$. It follows that $F^{\prime}$ is an inverse subsemigroup of $C(S)$. Clearly the identity of $F^{\prime}$ is $E(S)$, and so $F^{\prime}$ has group of units $\Sigma(S)$. Since the order on $F^{\prime}$ is just inclusion, and since $S$ is almost factorisable, it follows that for each element $[s]$ there exists $A \in U\left(F^{\prime}\right)$ such that $[s] \leq A$. Hence $F^{\prime}$ is factorisable. Clearly $S$ is isomorphic to $F^{\prime} \backslash U\left(F^{\prime}\right)$. We now turn to the general case. Put $F=\{[s]: s \in S\} \cup G$. The multiplication in $F$ extends that of $\{[s]: s \in S\}$ and $G$, and define $[s] g=[s] \alpha(g)$ and $g[s]=\alpha(g)[s]$. The multiplication is clearly well-defined, and associativity is easy to check. It follows that $F$ is an inverse monoid with group of units $G$. $F$ is factorisable, since $\cup \alpha(G)=S$ and $[s] \leq g$ if, and only if, $[s] \subseteq \alpha(g)$.

2. Semidirect product covers of almost factorisable semigroups. In [3, Corollary 4.9], McAlister constructs a functor from inverse semigroups to semidirect products of groups and semilattices and deduces that the almost factorisable semigroups are just the homomorphic images of semidirect products of groups and semilattices. We shall prove this latter result directly below. We begin, therefore, with some results on semidirect products of groups and semilattices.

Definition. If $\theta: S \rightarrow T$ is a homomorphism then there are well-defined functions $\theta_{e}: L_{e} \rightarrow L_{\theta(e)}$, for each idempotent $e \in E(S)$, obtained by restricting $\theta$ to $L_{e}$. A homomorphism $\theta: S \rightarrow T$ is said to be $\mathscr{L}$-injective (resp. $\mathscr{L}$-surjective) if all the functions $f_{e}$ are injective (resp. surjective). A homomorphism is said to be $\mathscr{L}$-bijective if it is both $\mathscr{L}$-injective and $\mathscr{L}$-surjective.

$\mathscr{L}$-injective homomorphisms are usually said to be idempotent pure or idempotent determined. It is easy to show that a homomorphism $\theta$ is $\mathscr{L}$-injective if and only if $\theta(x)$ an idempotent implies that $x$ is an idempotent.

Most of the following equivalences are well-known, but for the sake of completeness we prove them all.

Proposition 2.1. Let $S$ be an inverse semigroup. Then the following are equivalent.

(i) $S$ is strongly E-unitary.

(ii) $S$ is $E$-unitary and for all $a \in S$ and $e \in E(S)$ there exists $b \in S$ such that $e=b^{-1} b$ and $a^{-1} b \in E(S)$.

(iii) $S$ is E-unitary and almost factorisable.

(iv) The natural map $\sigma^{\natural}: S \rightarrow S / \sigma$ is $\mathscr{L}$-bijective.

(v) There is an $\mathscr{L}$-bijective homomorphism from $S$ to a group.

(vi) The function $\theta: S \rightarrow E(S) \times S / \sigma$ defined by $\theta(a)=\left(a^{-1} a, \sigma(a)\right)$ is a bijection.

(vii) The function $\phi: S \rightarrow E(S) \times S / \sigma$ defined by $\phi(a)=\left(a a^{-1}, \sigma(a)\right)$ is a bijection. 
Proof. (i) $\Rightarrow$ (ii). We shall suppose that $S=E \times G$ where $G$ is a group which acts on the meet semilattice $E$ by means of order automorphisms. It is clear that $S$ is $E$-unitary. Let $(e, g)$ be any element of $S$ and $(f, 1)$ any idempotent. Then the element $(g f, g)$ is such that $(g f, g)^{-1}(g f, g)=(f, 1)$ and $(e, g)^{-1}(f, g)=\left(g^{-1} e, g^{-1}\right)(f, g)=\left(g^{-1} e \wedge g^{-1} f, 1\right)$ an idempotent.

(ii) $\Rightarrow$ (iii). We shall show that $S$ is almost factorisable. Let $s \in S$. Then $s \in \sigma(s)$. By Theorem 1.2, $\sigma$ coincides with the compability relation on $S$ and so $\sigma(s)$ is a compatible subset of $S$. It is clear that $\sigma(s)$ is an order ideal. We shall therefore have established our claim if we show that $\sigma(s) \in \Sigma(S)$. Let $e \in E(S)$ be any idempotent. Then there exists an element $t$ such that $e=t^{-1} t$ and $s^{-1} t=f \in E(S)$. It follows that tos. But then $e \in \sigma(s)^{-1} \sigma(s)$. Thus $E(S)=\sigma(s)^{-1} \sigma(s)$. To show that $\sigma(s) \sigma(s)^{-1}=E(S)$, let $e \in E(S)$. By assumption there exists an element $u$ such that $e=u^{-1} u$ and $\left(s^{-1}\right)^{-1} u=i \in E(S)$. Thus $\sigma(s)=\sigma\left(u^{-1}\right)$. Put $v=u^{-1}$. Then $e=v v^{-1}$ and $s \sigma v$. Hence $\sigma(s) \sigma(s)^{-1}=E(S)$.

(iii) $\Rightarrow$ (iv). $\sigma^{\natural}$ is $\mathscr{L}$-injective. For suppose that $\sigma(s)=\sigma(t)$ and $s^{-1} s=t^{-1} t$. Then $s \sim t$ by Theorem 1.2. Thus by Lemma 1.1 , we have that $s=t$. Also, $\sigma^{\natural}$ is $\mathscr{L}$-surjective. For suppose that $e \in E(S)$ and $\sigma(s)$ is given. Then since $S$ is almost factorisable there exists $A \in \Sigma(S)$ that $s \in A$. Thus there exists $a \in A$ such that $a^{-1} a=e$. But $a \in A$ implies $a \sim s$ and so $\sigma(a)=\sigma(s)$.

(iv) $\Rightarrow$ (v). Immediate.

(v) $\Rightarrow$ (iv). Let $\theta: S \rightarrow G$ be an $\mathscr{L}$-bijective homomorphism to a group $G$. Then $\sigma \subseteq \operatorname{ker} \theta$ and it is immediate that $\sigma^{\natural}$ is $\mathscr{L}$-injective. To show that $\sigma^{\natural}$ is $\mathscr{L}$-surjective, let $s$ be any element of $S$ and $e$ any idempotent. There exists $t \in S$ such that $t^{-1} t=e$ and $\theta(t)=\theta(s)$. Now $\theta\left(s^{-1} t\right)$ is the identity of $G$, so that by the fact that $\theta$ is $\mathscr{L}$-injective we have that $s^{-1} t$ is an idempotent. Similarly, $s t^{-1}$ is an idempotent. Hence $s \sim t$ and so $s o t$.

(iv) $\Rightarrow$ (vi). Immediate.

(vi) $\Rightarrow$ (vii). Suppose $\phi(a)=\phi(b)$. Then $a a^{-1}=b b^{-1}$ and $\sigma(a)=\sigma(b)$. Clearly $\theta\left(a^{-1}\right)=\theta\left(b^{-1}\right)$. Thus $a^{-1}=b^{-1}$ and so $a=b$. Let $(e, \sigma(s)) \in E \times S / \sigma$. Then since $\theta$ is surjective there exists $t \in S$ such that $\theta(t)=\left(e, \sigma\left(s^{-1}\right)\right)$. Thus $t^{-1} t=e$ and $t \sigma s^{-1}$. Hence $t^{-1}$ is such that $t^{-1} \sigma s$ and $t^{-1}\left(t^{-1}\right)^{-1}=e$. Thus $\phi\left(t^{-1}\right)=(e, \sigma(s))$.

(vii) $\Rightarrow$ (vi). A similar argument to (vi) $\Rightarrow$ (vii).

(vii) $\Rightarrow$ (i). We shall use the fact that both the functions $\phi$ and $\theta$ defined above are bijections. Observe first that $S$ is $E$-unitary. For suppose that $e \leq a$ where $e$ is an idempotent. Then $e=a e$. Thus $\sigma(e)=\sigma(a) \sigma(e)=\sigma(a)$. It follows that $\theta(a)=\theta\left(a^{-1} a\right)$ and so $a=a^{-1} a$. We now define an action of $S / \sigma$ on $E(S)$ in such a way that the semidirect product of $E(S)$ and $S / \sigma$ is isomorphic, via $\phi$, to $S$. Put $\sigma(s) . e=t t^{-1}$ where $\theta(t)=(e, \sigma(s))$. We check the two defining properties (1) and (2) below for an action.

(1): if $\sigma(e)$ is the identity of $S / \sigma$ then $\theta(e)=(e, \sigma(e))$ and so $\sigma(e) . e=e$.

(2): $\sigma(u)(\sigma(v) \cdot e)=\sigma(u) \cdot a a^{-1}$ where $\theta(a)=(e, \sigma(v))$, and $\sigma(u) \cdot a a^{-1}=b b^{-1}$ where $\theta(b)=\left(a a^{-1}, \sigma(u)\right)$. Thus

$$
a^{-1} a=e, \quad a \sigma v, \quad b^{-1} b=a a^{-1} \text { and } b \sigma u .
$$

Now

$$
\theta(b a)=\left((b a)^{-1} b a, \sigma(b a)\right)=\left(a^{-1} a, \sigma(u v)\right)=(e, \sigma(u v))
$$

Thus

$$
\sigma(u v) \cdot e=(b a)(b a)^{-1}=b b^{-1}=\sigma(u)(\sigma(v) \cdot e) .
$$

Hence $\sigma(u v) . e=\sigma(u)(\sigma(v) . e)$. 
We claim that $S / \sigma$ acts on $E(S)$ by means of order isomorphisms. Let $e \leq f$. Then $\sigma(a) \cdot e=u u^{-1}$ and $\sigma(a) \cdot f=v v^{-1}$ where

$$
\theta(u)=(e, \sigma(a)) \text { and } \theta(v)=(f, \sigma(a)) .
$$

But $e \leq f$ means $u^{-1} u \leq v^{-1} v$, and $u \sigma v$ and so $u \sim v$ since $S$ is $E$-unitary. Thus by Lemma 1.1 , we have that $u \leq v$ and so $u u^{-1} \leq v v^{-1}$. Thus $\sigma(a) . e \leq \sigma(a) . f$.

Now suppose that $\sigma(a) . e \leq \sigma(a) . f$. Then $\sigma(a) \cdot e=u u^{-1}$ and $\sigma(a) \cdot f=v v^{-1}$ where

$$
\theta(u)=(e, \sigma(a)) \text { and } \theta(v)=(f, \sigma(a)) .
$$

But $u \sigma v$ and $u u^{-1} \leq v v^{-1}$ implies by Lemma 1.1 that $u \leq v$. Hence $e \leq f$.

It only remains to prove that $\phi$ is a homomorphism. Now

$$
\phi(a) \phi(b)=\left(a a^{-1}, \sigma(a)\right)\left(b b^{-1}, \sigma(b)\right)=\left(a a^{-1} a \wedge \sigma(a) \cdot b b^{-1}, \sigma(a b)\right) .
$$

But $\sigma(a) \cdot b b^{-1}=t t^{-1}$ where $\theta(t)=\left(b b^{-1}, \sigma(a)\right)$. Thus

$$
\phi(a) \phi(b)=\left(a a^{-1} t t^{-1}, \sigma(a b)\right),
$$

whereas

$$
\phi(a b)=\left(a b(a b)^{-1}, \sigma(a b)\right) .
$$

By Theorem 1.2, toa implies that $t \sim a$, so that, again by Theorem $1.2, t t^{-1} a=a t^{-1} t$. Hence $a^{-1} t t^{-1}=t^{-1} t a^{-1}$ and so $a a^{-1} t t^{-1}=a t^{-1} t a^{-1}$. But $t^{-1} t=b b^{-1}$. Thus $a a^{-1} t t^{-1}=$ $a b b^{-1} a^{-1}=a b(a b)^{-1}$. Hence $\phi$ is a homomorphism and so an isomorphism.

The equivalence of (i) and (ii) is the dual of [3, Theorem 4.12].

Lemma 2.2. Let $S$ be strongly E-unitary. Then $\Sigma(S)=\{\sigma(s): s \in S\}$ and the groups $\Sigma(S)$ and $S / \sigma$ are the same.

Proof. Let $A \in \Sigma(S)$ and $s \in A$. Then since $A$ is a compatible subset of $S$ we have that $A \subseteq \sigma(s)$. Let $t \sigma s$. Now $t^{-1} t \in A^{-1} A$ and so there exists $a \in A$ such that $a^{-1} a=t^{-1} t$. But $\sigma(a)=\sigma(t)$ and $a^{-1} a=t^{-1} t$ and so by Proposition 2.1(vi), we have that $a=t$. Thus $A=\sigma(s)$. On the other hand, given a $\sigma$-class $\sigma(s)$ it is a permissible subset of $S$ because $S$ is $E$-unitary. Furthermore, $\sigma(s)^{-1} \sigma(s)=E(S)$. For let $e \in E(S)$. Then by Proposition 2.1, there exists $t \in S$ such that $e=t^{-1} t$ and $s \sigma t$. A dual argument establishes that $\sigma(s) \sigma(s)^{-1}=$ $E(S)$. To conclude, we have to show that the multiplications in $\Sigma(S)$ and $S / \sigma$ agree. It is clear that as subsets, $\sigma(s) \sigma(t) \subseteq \sigma(s t)$. We shall now show that the reverse inclusion holds. Let $x$ ost. Since $x x^{-1} \in E(S)$ there exists $u \in \sigma(s)$ such that $u u^{-1}=x x^{-1}$. Thus $u^{-1} x \sigma s^{-1} s t$, and so $u^{-1} x \sigma t$. Put $v=u^{-1} x$. Then $v \in \sigma(t)$. Hence $x=u v \in \sigma(s) \sigma(t)$.

In the result below, we construct a family of strongly $E$-unitary covers of an almost factorisable semigroup.

Proposition 2.3. Let $S$ be an almost factorisable semigroup, and let $\alpha: G \rightarrow \Sigma(S)$ be a group homomorphism such that $\cup \alpha(G)=S$. Define $P(S, G, \alpha)$ to be the set

$$
P(S, G, \alpha)=\{(s, g) \in S \times G: s \in \alpha(g)\},
$$

equipped with the direct product multiplication. Define $\pi_{1}: P(S, G, \alpha) \rightarrow S$ by $\pi_{1}(s, g)=s$ and $\pi_{2}: P(S, G, \alpha) \rightarrow G$ by $\pi_{2}(s, g)=g$. Then $P(S, G, \alpha)$ is an inverse semigroup, $\pi_{1}$ is a surjective, idempotent separating homomorphism and $\pi_{2}$ is a surjective, L्L-bijective homomorphism. In particular, $P(S, G, \alpha)$ is strongly E-unitary. 
Proof. We check closure first. Let $(s, g),(t, h) \in P(S, G, \alpha)$. Then $s \in \alpha(g)$ and $t \in \alpha(h)$. Thus $s t \in \alpha(g) \alpha(h)=\alpha(g h)$, and so $(s t, g h) \in P(S, G, \alpha)$. A simple argument shows that $P(S, G, \alpha)$ is closed under inverses. It is clear that $\pi_{1}$ is an idempotent separating homomorphism, furthermore it is surjective because for each $s \in S$ there is a $g \in G$ such that $s \in \alpha(g)$. It is clear that $\pi_{2}$ is a surjective homomophism. We now show that $\pi_{2}$ is $\mathscr{L}$-bijective. Suppose that $(s, g) \mathscr{L}(t, h)$ and $\pi_{2}(s, g)=\pi_{2}(t, h)$. Then $g=h, s$, $t \in \alpha(g)$ and $s \mathscr{L} t$. But $\alpha(g)$ is a permissible subset of $S$, so that $s=t$ by Lemma 1.3. Thus $\pi_{2}$ is $\mathscr{L}$-injective. Now let $g \in G$ and $(e, 1) \in E(P(S, G, \alpha))$. Then $e \in \alpha(g)^{-1} \alpha(g)$, and so there exists $s \in \alpha(g)$ such that $s^{-1} s=e$. Thus $(s, g) \in P(S, G, \alpha),(s, g)^{-1}(s, g)=(e, 1)$ and $\pi_{2}(s, g)=g$. Hence $\pi_{2}$ is $\mathscr{L}$-surjective, and so, by Proposition $2.1, P(S, G, \alpha)$ is strongly $E$-unitary.

We may now describe the relationship between almost factorisable semigroups and strongly $E$-unitary semigroups.

THEOREM 2.4. (i) Homomorphic images of almost factorisable semigroups are almost factorisable.

(ii) Every almost factorisable inverse semigroup is an idempotent separating homomorphic image of a strongly E-unitary semigroup.

(iii) The class of almost factorisable semigroups is the closure under homomorphisms of the class of strongly E-unitary semigroups.

Proof. (i) Let $\theta: S \rightarrow T$ be a surjective homomorphism from an almost factorisable semigroup $S$ to a semigroup $T$. Let $t \in T$ and let $s \in S$ such that $\theta(s)=t$. Since $S$ is almost factorisable there exists $A \in \Sigma(S)$ such that $s \in A$. By Proposition 1.5, $\theta(A) \in \Sigma(T)$, since $\theta$ is full, and $t \in \theta(A)$.

(ii) Let $T$ be an almost factorisable semigroup. By Proposition 2.3, we obtain a strongly $E$-unitary cover by taking $G=\Sigma(T)$ and $\alpha$ to be the identity map.

(iii) We have seen that strongly $E$-unitary semigroups are almost factorisable by Proposition 2.1. It follows from (i), that all homomorphic images of such semigroups are almost factorisable. But from (ii), every almost factorisable semigroup is a homomorphic image of a strongly $E$-unitary semigroup.

We now show how to construct all strongly $E$-unitary covers of an almost factorisable inverse semigroup, thus completing the analysis begun in Proposition 2.3.

THEOREM 2.5. Let $S$ be an almost factorisable semigroup, and let $\phi: P \rightarrow S$ be an idempotent separating cover of $S$ where $P$ is strongly $E$-unitary. Then there exists a group homomorphism $\phi: P / \sigma \rightarrow \Sigma(S)$ such that $\cup \phi(P / \sigma)=S$, and an isomorphism $\psi: P \rightarrow$ $P(S, P / \sigma, \phi)$ such that $\pi_{1} \psi=\phi$.

Proof. Since $\phi$ is surjective, it is full, and so induces a homomorphism from $\Sigma(P)$ to $\Sigma(S)$ by Proposition 1.5, which we shall also denote by $\phi$. By Lemma 2.2 , we have that $P / \sigma=\Sigma(P)$ as groups, so we have a homomorphism $\phi: P / \sigma \rightarrow \Sigma(S)$. Let $s \in S$. Then there exists $p \in P$ such that $\phi(p)=s$, and so $s \in \phi(\sigma(p))$. Hence $\cup \phi(P / \sigma)=S$. Thus we can form the semigroup $P(S, P / \sigma, \phi)$ according to Proposition 2.3. Define

$$
\psi: P \rightarrow P(S, P / \sigma, \phi) \text { by } \psi(p)=(\phi(p), \sigma(p)) .
$$

This is a well-defined function, for $\phi(p) \in \phi(\sigma(p))$. It is clear that $\psi$ is a homomorphism. 
$\psi$ is injective. For suppose that $\psi(p)=\psi(q)$. Then $(p, q) \in \operatorname{ker} \phi \cap \sigma$. Since $\phi$ is idempotent separating $p \mathscr{L} q$. But $P$ is $E$-unitary, so that by Theorem 1.2 and $p \sigma q$, we obtain $p \sim q$. Thus by Lemma 1.1 ; we have that $p=q$. Finally, $\psi$ is surjective. For if $(s, \sigma(p)) \in P(S, P / \sigma, \phi)$ then $s \in \phi(\sigma(p))$. Thus there is $q \in \sigma(p)$ such that $\phi(q)=s$. But then $\psi(q)=(\phi(q), \sigma(q))=(s, \sigma(p))$. It follows that the cover $\phi: P \rightarrow S$ is isomorphic to the cover $\pi_{1}: P(S, P / \sigma, \phi) \rightarrow S$ in the sense that $\pi_{1} \psi=\phi$.

3. Arbitrary inverse semigroups and almost factorisable semigroups. In this section, we investigate the relationship between arbitrary inverse semigroups and almost factorisable semigroups. In particular, we shall make precise an embedding first obtained by McAlister [3], [5].

Definition. A McAlister triple is a triple $(G, X, Y)$ satisfying the following conditions:

(MT1) $G$ is a group and $X$ a poset on which $G$ acts (on the left) by order automorphisms.

(MT2) $Y$ is an order ideal and subsemilattice of $X$.

(MT3) For each $x \in X$ there exists $y \in Y$ such that $y \leq x$.

(MT4) $G Y=X$.

Associated with every McAlister triple $(G, X, Y)$ is an $E$-unitary semigroup $P=P(G, X, Y)$ with underlying set

$$
P=\left\{(y, g) \in Y \times G: g^{-1} y \in Y\right\} .
$$

Such semigroups are called $P$-semigroups. We refer the reader to Petrich [7] for details.

It can be shown that every $E$-unitary inverse semigroup is isomorphic to a $P$-semigroup [7]. Observe that the inverse semigroup $P=P(G, X, Y)$ is finite if, and only if, $G$ and $X$ are finite. For suppose $P$ is finite. Then $G$ and $Y$ are finite, but $G Y=X$ so that $X$ is finite. The converse is immediate.

Definition. An $E$-unitary semigroup is said to be over a semilattice if it is isomorphic to a $P$-semigroup of the form $P(G, X, Y)$ where $X$ is also a meet semilattice. An $F$-inverse monoid is an $E$-unitary monoid which is over a semilattice.

$E$-unitary semigroups over semilattices were introduced by McAlister [4]. An abstract characterisation of $E$-unitary semigroups over semilattices is given below, see [4, Theorem 2.3] for a proof.

THEOREM 3.1. Let $S$ be an E-unitary inverse semigroup. Then $S$ is over a semilattice if, and only if, for all $e, f \in E(S)$ and $s \in S$ the set $\sigma(s) \cap e S f$ has a maximum element.

The proof of (i) below is a standard result in inverse semigroup theory, consult Petrich [7]. McAlister [4] contains a proof of (ii) below (see also Reilly and Munn [8]).

THEOREм 3.2. (i) Every inverse semigroup has an E-unitary cover. If the semigroup is finite then it has a finite E-unitary cover.

(ii) Every inverse semigroup has an E-unitary cover over a semilattice.

(iii) Every inverse monoid has an F-inverse cover.

It appears to be unknown whether every finite inverse semigroup has a finite $E$-unitary cover over a semilattice. See [9, Problem 3.7, page 462]. 
Terminology. Throughout this section, all $E$-unitary covers will be over semilattices, unless stated otherwise.

We shall show that the problem of finding all $E$-unitary covers of a semigroup $S$ can be divided into two basic steps. The first step is connected with the notion of enlargement defined below.

Definition. Let $\iota: S \rightarrow T$ be an embedding. Then $T$ is said to be an enlargement of $S$ if the following conditions hold.

(E1) $E(\iota(S))$ is an order ideal of $T$.

(E2) If $x \in T$ and $x^{-1} x, x x^{-1} \in E(\iota(S))$ then $x \in \iota(S)$.

(E3) For each $e \in E(T)$ there exists $f \in E(\iota(S))$ such that $e \mathscr{D} f$.

We shall often assume that $\iota$ is subsemigroup inclusion.

The conditions above first appeared together in [2]. We were led to formulate them by considering the semigroup analogue of Ehresmann's Maximum Enlargement Theorem which is discussed in [1]. Conditions (E1) and (E2) were mentioned by McAlister who calls subsemigroups satisfying these conditions alone heavy [3], [5]. The final condition, (E3), is mentioned in a remark in [3, Section 6.1.2], but is not used in the paper as a whole.

The result below is proved in [2]. McAlister [3] had already noted that $P$ was a heavy subsemigroup of $\Pi$.

Proposition 3.3. Let $P=P(G, X, Y)$ be a P-semigroup over a semilattice $X$. Put $\Pi=P(G, X, X)$, the semidirect product of $G$ and $X$. Then $\Pi$ is an enlargement of $P$.

A stronger form of the above result is (ii) below.

Proposition 3.4. (i) Let $S$ be strongly E-unitary. Then $C(S)$ is factorisable.

(ii) $S$ is E-unitary over a semilattice if, and only if, $S$ has a strongly E-unitary enlargement.

Proof. (i) Immediate from the representation of $S$ as a semidirect product of a group and a semilattice.

(ii) Suppose that $S$ has a strongly $E$-unitary enlargement $T$; to ease notation we shall suppose that $S$ is a subsemigroup of $T$. Let $e, f \in E(S)$ and $s \in S$. We shall show that $e S f \cap \sigma(s)$ has a maximum element; the result will then follow by Theorem 3.1. It is clear that $\sigma(s) \in C(T)$, for $\sigma(s)$ is a compatible subset of $S$ and so of $T$; also it is an order ideal of $S$ and $S$ is an order ideal of $T$ and so $\sigma(s)$ is an order ideal of $T$. By (i) there exists $A \in \Sigma(T)$ such that $\sigma(s) \subseteq A$. Thus there exists $x \in A$ such that $x^{-1} x=f$. Put $y=e x$. Then $y \in A$ since $A$ is an order ideal. Now $y^{-1} y \leq f$ and $y y^{-1} \leq e$ so that $y^{-1} y, y y^{-1} \in E(S)$, by (E1). Thus $y \in S$ by (E2). Hence $y \in e S f$. But $y, s \in A$ and so $y \sim s$. Thus $y \sigma s$ in $S$. We claim that $y$ is the maximum element of $e S f \cap \sigma(s)$. Let $z \in e S f \cap \sigma(s)$. Since $z f=z$ we have that $z^{-1} z \leq f$. Thus by Lemma 1.1, $z \leq x$. But then $z=e z \leq e x \leq y$. The converse is immediate by Proposition 3.3.

The proof of the following is straightforward.

Proposition 3.5. Let $T$ be an enlargement of $S$ and let $\theta$ be a surjective idempotent separating homomorphism $\theta: T \rightarrow W$. Then $W$ is an enlargement of $\theta(S)$. 
The proof of the following result appears in [2]. Theorem 3.6 of [3] is a special case.

Proposition 3.6. Let $T$ be an enlargement of $S$. Then every idempotent separating congruence $\rho$ on $S$ extends to a unique idempotent separating congruence $\tau$ on $T$ such that $\tau \cap(S \times S)=\rho$.

Proposition 3.7. (i) Let $S \subseteq T \subseteq U$ be inverse semigroups. If $T$ is an enlargement of $S$ and $U$ is an enlargement of $T$ then $U$ is an enlargement of $S$.

(ii) Let $V \subseteq T$ and $\theta: S \rightarrow T$ a surjective, idempotent separating homomorphism. Suppose that $T$ is an enlargement of $V$, then $S$ is an enlargement of $U=\theta^{-1}(V)$.

Proof. (i) Straightforward.

(ii) Clearly, $U$ is an inverse subsemigroup of $S$. Now show (E1) holds. Let $s \leq u$ where $\theta(u) \in V$. Then $\theta(s) \leq \theta(u) \in V$. But $T$ is an enlargement of $V$ so that $\theta(s) \in V$. Thus $s \in U$. Next show (E2) holds. Let $s \in S$ such that $s^{-1} s, s s^{-1} \in U$. Then $\theta(s)^{-1} \theta(s)$, $\theta(s) \theta(s)^{-1} \in V$. Hence $\theta(s) \in V$ and so $s \in U$. Finally, (E3) holds. Let $e \in E(S)$; then $\theta(e) \in T$. Thus there exists $t \in T$ such that $t t^{-1}=\theta(e)$ and $t^{-1} t \in E(V)$. Let $s \in \theta^{-1}(t)$. Then $\theta\left(s s^{-1}\right)=t t^{-1}=\theta(e)$. But $\theta$ is idempotent separating and so $s s^{-1}=e$. Also $\theta\left(s^{-1} s\right)=t^{-1} t \in E(V)$. Thus $s^{-1} s \in U$.

The following theorem establishes a close link between $E$-unitary covers of a semigroup $S$ over semilattices and almost factorisable enlargements of $S$.

THEOREM 3.8. (i) Let $F$ be an almost factorisable enlargement of $S$ and let $\beta: G \rightarrow \Sigma(F)$ be a group homomorphism such that $\cup \beta(G)=F$. Put

$$
P=P(F, G, \beta, S)=\{(s, g) \in S \times G: s \in \beta(g)\} .
$$

Then with componentwise multiplication $P$ is an E-unitary semigroup over a semilattice, $\pi_{1}: P \rightarrow S$ is a surjective, idempotent separating homomorphism, and $\pi_{2}: P \rightarrow G$ is a surjective, $\mathscr{L}$-injective homomorphism.

(ii) Let $P=P(G, X, Y)$ be an E-unitary cover over a semilattice of the inverse semigroup $S$ by the idempotent separating homomorphism $\theta: P \rightarrow S$ and let $\Pi=P(G, X$, $X)$. Then there is an almost factorisable enlargement $T$ of $S$ and a group homomorphism $\alpha: \Pi / \sigma \rightarrow \Sigma(T)$ with $\cup \alpha(\Pi / \sigma)=T$, such that $P$ is isomorphic to $P(T, \Pi / \sigma, \alpha, S)$ by means of an isomorphism $\psi: P \rightarrow P(T, \Pi / \sigma, \alpha, S)$ with $\theta=\pi_{1} \psi$.

Proof. (i) By Proposition 2.3, $P(F, G, \beta)$ is strongly $E$-unitary and $\pi_{1}: P(F, G$, $\beta) \rightarrow F$ is a surjective, idempotent separating homomorphism. It is easy to see that $\pi_{1}^{-1}(S)=P(F, G, \beta, S)$. By Proposition 3.7(ii), $P(F, G, \beta)$ is an enlargement of $P(F, G$, $\beta, S)$. By Proposition 3.4(ii), $P(F, G, \beta, S)$ is an $E$-unitary semigroup over a semilattice. The rest of part (i) is now immediate.

(ii) By Proposition 3.3, $\Pi=P(G, X, X)$ is an enlargement of $P$. Since ker $\theta$ is an idempotent separating congruence on $P$ there exists, by Proposition 3.6, a unique idempotent separating congruence $\rho$ on $\Pi$ such that $\rho \cap(P \times P)=\operatorname{ker} \theta$. Put $T=\Pi / \rho$. By Theorem 2.4, $T$ is almost factorisable. Put $S^{\prime}=\rho^{\natural}(P)$. Clearly, $S$ and $S^{\prime}$ are isomorphic by means of an isomorphism $\zeta: S \rightarrow S^{\prime}$ given by $\zeta(\theta(p))=\rho^{\natural}(p)$. From now on we shall identify $S$ and $S^{\prime}$ by means of $\zeta$. By Proposition 3.5, $T$ is an enlargement of $S^{\prime} . \Pi$ is a strongly $E$-unitary cover of $T$, thus, by Theorem 2.5 , there is a group homomorphism $\alpha: \Pi / \sigma \rightarrow \Sigma(T)$ such that $\cup \alpha(\Pi / \sigma)=T$, where $\alpha(\sigma(p))=\rho^{\natural}(\sigma(p))$. 
There is an isomorphism $\psi: \Pi \rightarrow P(T, \Pi / \sigma, \alpha)$ defined by $\psi(p)=\left(\rho^{\natural}(p), \sigma(p)\right)$ such that $\pi_{1} \psi=\rho^{q}$. We claim that $\psi$ induces an isomorphism from $P(G, X, Y)$ to $P(T, \Pi / \sigma$, $\left.\alpha, S^{\prime}\right)$. If $p \in P$ then $\rho^{\natural}(p) \in S^{\prime}$. Thus $\psi$ induces a homomorphism from $P(G, X, Y)$ to $P\left(T, \Pi / \sigma, \alpha, S^{\prime}\right)$. On the other hand let $\left(\rho^{4}(p), \alpha(\sigma(q))\right) \in P\left(T, \Pi / \sigma, \alpha, S^{\prime}\right)$. Then $p \in \sigma(q)$ and $p \in P$. Thus $\left(\rho^{q}(p), \alpha(\sigma(q))\right)=\psi(p)$. Hence $\psi$ induces an isomorphism from $P(G, X, Y)$ to $P\left(T, \Pi / \sigma, \alpha, S^{\prime}\right)$.

We may now describe a two step method for constructing all $E$-unitary covers over semilattices of an inverse semigroup $S$.

(Step 1) Construct all almost factorisable enlargements $F$ of $S$.

(Step 2) For each such enlargement $F$ construct all group homomorphisms $\alpha: G \rightarrow$ $\Sigma(F)$ such that $\cup \alpha(G)=F$.

Then $\pi_{1}: P(F, G, \alpha, S) \rightarrow S$ is an $E$-unitary cover over a semilattice, and every $E$-unitary cover of $S$ over a semilattice is isomorphic to one constructed in this way.

This approach to $E$-unitary covers leads to a reformulation of the question of the existence of finite covers over semilattices.

Proposition 3.9. Let $F$ be an inverse semigroup and let e be an idempotent in $F$. Then $F$ is an enlargement of eFe if, and only if, $F=F e F$.

Proof. Observe first that $e F e$ always satisfies (E1) and (E2). Suppose that $F$ is an enlargement of $e F e$, and let $x \in F$. Then there exist elements $a$ and $b$ of $F$ such that

$$
a a^{-1}=x x^{-1}, x^{-1} x=b b^{-1} \text { and } a^{-1} a, b^{-1} b \in E(e F e) .
$$

But then $x=a\left(a^{-1} x b\right) b^{-1}$ and $a^{-1} x b \in e F e$. Thus $x=a e\left(a^{-1} x\right) \in F e F$. Conversely, suppose that $F=F e F$, and $f \in E(F)$. Then $f=x e y$ for some $x, y \in F$. By Lemma 1.4, $f=a i b$ where the product on the right hand side is a trace product, $a \leq x, i \leq e$ and $b \leq y$. It is now clear that $f \mathscr{D} i$.

Proposition 3.10. For every inverse monoid $S$ there exists an almost factorisable semigroup $F$ and an idempotent $e \in F$ such that $S$ is isomorphic to eFe and $F=F e F$.

Proof. Immediate by Theorem 3.2(iii), Theorem 3.8 and Proposition 3.9.

We now have the following reformulation of the existence of finite $F$-inverse covers of finite inverse monoids.

Proposition 3.11. A finite inverse monoid $S$ has a finite $F$-inverse cover if, and only if, $S$ has a finite almost factorisable enlargement.

Proof. Suppose that $S$ has a finite $F$-inverse cover. Then by Theorem 3.8, $S$ has a finite almost factorisable enlargement. Conversely, suppose that $S$ is isomorphic to $e F e$, $F=F e F$ and $F$ is finite and almost factorisable. Without loss of generality, we may assume that $S=e F e$. Then again by Theorem 3.8, we may construct a finite $F$-inverse cover of $S$, namely $P(F, \Sigma(F), \iota, S)$ where $\iota$ is the identity map on $\Sigma(F)$.

\section{REFERENCES}

1. M. V. Lawson, The geometric theory of inverse semigroups II: $E$-unitary covers of inverse semigroups, J. Pure and Applied Algebra 83 (1992), 121-139. 
2. M. V. Lawson, An equivalence theorem for inverse semigroups, Semigroup Forum 47 (1993), 7-14.

3. D. B. McAlister, Some covering and embedding theorems for inverse semigroups, $J$. Australian Math. Soc. 22 (Series A) (1976), 188-211.

4. D. B. McAlister, E-unitary inverse semigroups over semilattices, Glasgow Math. J. 19 (1978), 1-12.

5. D. B. McAlister, Some covering theorems for locally inverse semigroups, J. Australian Math. Soc. 39 (Series A) (1985), 63-74.

6. K. S. S. Nambooripad, The natural partial order on a regular semigroup, Proc. Edinburgh Math. Soc. 23 (1980), 249-260.

7. M. Petrich, Inverse semigroups (Wiley, 1984).

8. N. R. Reilly and W. D. Munn, E-unitary congruences on inverse semigroups, Glasgow Math. J. 17 (1976), 57-75.

9. J. Rhodes (editor), Monoids and semigroups with applications (World Scientific, 1991).

10. B. Schein, Completions, transiational hulls and ideal extensions of inverse semigroups, Czechoslovak Math. J. 23 (1973), 575-610.

University College of North Wales, SCHOOL OF Mathematics, DEAN STREET,

BANGOR, GWYNEDD, LL57 1UT.

CYMru/WaLES U.K. 